\title{
DISTÚRBIOS COGNITIVOS NA DOENÇA DE PARKINSON
}

\author{
CORRELAÇÕES ELETRENCEFALOGRÁFICAS
}

\author{
JOÃO SANTOS PEREIRA*, MARIA LÚCIA VELLUTINI PIMENTEL **, PAULO LUCIANO H. GOMES***
}

\begin{abstract}
RESUMO - 62 pacientes com diagnóstico de doença de Parkinson idiopática e 30 pacientes controle foram avaliados quanto a distúrbios mnemônicos e psíquicos, utilizando-se os critérios do DSM III-R, o mini-mental e a escala de Hamilton para depressão. Todos foram submetidos a eletrencefalograma (EEG) com fotoestimulação intermitente. Dos pacientes parkinsonianos, $24,2 \%$ apresentavam depressão psíquica, 14,5\% ansiedade, $12,9 \%$ distimia, 3,2\% demência e 45,2\% nāo apresentavam quaisquer alteraçðes de acordo com os critérios dos DSM III-R. Nos EEG evidenciavam-se ondas teta bilaterais com predomínio fronto-temporal ou temporal, com maior comprometimento a esquerda, em $58,1 \%$ desses pacientes. Em apenas $16(7 \%)$ dos pacientes controle tais achados foram observados, porém, em nenhum dos dois grupos ocorreram alteraçðes no EEG pela fotoestimulação. As alteraçōes do EEG foram estatisticamente significantes quando se compararam pacientes parkinsonianos com pacientes controle ( $p<0,001)$; o mesmo não ocorreu quando se compararam pacientes parkinsonianos que apresentavam distúrtio psíquico com aqueles que não o apresentavam.
\end{abstract}

PALAVRAS-CHAVE: doença de Parkinson, depressão, distúrbios cognitivos, alteraçōes cletrencefalograficas.

\section{Mental impairment in Parkinson disease: clinical and electroencephalographic correlations}

SUMMARY - Sixty-two patients with idiopathic Parkinson disease and 30 patients of a control group were clinically evaluated in the light of cognitive and/or psychic impairments according to DSM III-R, mini-mental state examination and Hamilton rating scale for depression. These patients were also submitted to electroencephalogram registration (EEG) with photic stimulation. From the parkinsonian group, 45.2\% did not have mental manifestations as classified in the DSM III-R. Other $24.2 \%$ had depression. $14.5 \%$ had anxiety, $12,9 \%$ had dysthymic disorder and $3.2 \%$ had dementia. Considering the EEG, $58.1 \%$ of the parkinsonian patients had theta waves bilaterally, with predominance in frontal temporal or temporal areas, though more frequent on the left hemisphere than on the right one. Only $16.7 \%$ of the patients from the control group had these same findings in the EEG but neither of the two groups had their EEG modified by photic stimulation. The EEG findings were statistically significant when both groups were compared. However, these findings were not significant comparing parkinsonian patients with psychic impairment with the ones who did not have such impairments.

KEY WORDS: Parkinson disease, depression, cognitive impairment, electroencephalographic findings.

Na descrição original da doença que leva o seu nome, James Parkinson (1817) não menciona comprometimento intelectual e/ou cognitivo nesses pacientes. Somente no final do século XIX

Estudo realizado no Ambulatório de Distúrbios do Movimento da Disciplina de Neurologia da Faculdade de Ciências Médicas (FCM) da Universidade do Estado do Rio de Janeiro (UERJ) com o apoio CNPq: *Professor Adjunto da FCM-UERJ, Doutor em Neurologia pela Escola Paulista de Medicina; **Médica Neurologista; ***Professor Auxiliar-Ensino da FCM-UERJ, responsável pelo setor de eletrencefalografia. Aceite: 8-julho-1994.

Dr. João Santos Pereira - Serviço de Neurologia, Hospital Universitário Pedro Emesto - Rua 28 de setembro 77/ $2^{\circ}$ andar - 20551-030 Rio de Janeiro RJ - Brasil. 
surgiram alguns artigos relatando alteraçōes mentais e de personalidade na doença de Parkinson. Alguns desses autores (Ball, 1882; Parant, 1883; Compin, 1902; Malie, 1908) fizeram referência à irascibilidade, comprometimento de memória, melancolia e demência nesses pacientes ${ }^{17}$. Em 1949 , Mjones observou distúrbios mentais do tipo reativo e orgânico, com predomínio desse último, em $40 \%$ dos pacientes com doença de Parkinson avaliados ${ }^{15}$. Tune e col. encontraram alta incidência de depressão, psicose e distúrbios cognitivos em pacientes parkinsonianos ${ }^{23}$. Eles também concluiram haver relação entre doença de Parkinson e depressão, embora não existisse unanimidade quanto à associaçāo entre a gravidade da doença e a incidência e severidade da depressão" ${ }^{1 !}$. Alguns autores basearam-se nessa correlação para dar apoio à teoria das alterações das catecolaminas no locus ceruleus $^{19}$. Na doença de Parkinson idiopática parece ocorrer degeneração do sistema mesocortical dopaminérgico, pois a atividade da tirosina hidroxilase e os níveis de catecolaminas estāo diminuidos no tegmento mesencefálico ventral ${ }^{6,10}$. Acredita-se que os niveis reduzidos de dopamina no córtex occipital na doença de Parkinson possam ser evidenciados pela eletrencefalografia (EEG) através da fotoestimulaçāo intermitente ${ }^{4}$.

Com essas observações e com os conhecimentos de que as mudanças no traçado eletrencefalográfico podem ser encontradas em vários distúrbios mentais dos idosos, resolvemos elaborar este estudo, correlacionando as alterações cognitivas dos pacientes parkinsonianos e os achados de EEG.

\section{MATERIAL E MÉTODOS}

Foram avaliados 62 pacientes caracterizados clínica e radiologicamente, através de tomografia computadorizada cerebral, como tendo doença de Parkinson idiopática, independentemente de sexo, raça, tempo de doença e medicaçāo utilizada, com idade variando de 42 a 81 anos e estando a maioria dos pacientes na faixa etária compreendida entre 51 e 70 anos (apenas um tinha idade abaixo de 50 anos) no periodo de 1989 a 1992 , no Ambulatório de Distúrbios do Movimento, da Disciplina de Neurologia do Hospital Universitário Pedro Ernesto da Universidade do Estado do Rio de Janeiro (HUPE/UERJ).

O grupo controle foi composto de 30 pacientes com idade variando de 50 a 79 anos, escolhidos aleatoriamente entre os pacientes de ambulatório que procuravam espontaneamente o hospital e que não apresentavam doenças neurológicas, cardiovasculares, traumatismo cranioencefálico, distúrbios metabólicos e que não faziam uso de qualquer medicação que pudesse interferir com a atividade cortical normal, assim como nāo tinham história familiar de doença de Parkinson.

Todos os pacientes foram submetidos individualmente a avaliação de distúrbios psíquicos e depressão utilizando-se o mini-mental, a escala de Hamilton para depressão e os critérios do DSM III-R, sendo considerado para o mini-mental o valor mínimo de 24 para a normalidade.

Em seguida, em todos os pacientes foi realizado EEG com fotoestimulaçāo intermitente, utilizando-se aparelho Alvar TR XVI com 12 canais, com mimeógrafo e fotoestimulador. Os pacientes, após tomarem ciência do cstudo em andamento e concordarem com a metodologia empregada, foram orientados a suspender a medicação dopaminérgica 24 horas antes da realização do exame e a procurar o Serviço ou retomar a medicação no caso de piora do quadro clínico e/ou outra intercorrência.

\section{RESULTADOS}

Dos 62 pacientes parkinsonianos avaliados, $35(56,4 \%)$ apresentaram alteraçōes do EEG caracterizadas principalmente pelo aparecimento de ondas pontiagudas e bilaterais, variando de $6 \mathrm{a}$ $15 \mathrm{~Hz}$, com predomínio em áreas fronto-temporais, ou ondas teta de predomínio nessas regiões, sendo o hemisfério esquerdo mais comprometido que o direito. Esses traçados não se modificaram com a fotoestimulação intermitente.

$\mathrm{Na}$ avaliação pelo mini-mental verificamos que 21 pacientes $(33,8 \%)$ apresentaram valores inferiores a 24, valor mínimo considerado para distúrbio mnemônico. Na avaliaçăo pela escala de 
Tabela J. Percentagem de alteraçōes eletrencefalográficas encontradas nos pacientes parkinsonianos e no grupo controle.

\begin{tabular}{lcc}
\hline Pacientes & \multicolumn{2}{c}{ Eletrencefalograma } \\
\hline & com alteração & sem alteração \\
\hline $\begin{array}{c}\text { Parkinsonianos } \\
(\mathrm{n}=62)\end{array}$ & $35(56,4 \%)$ & $27(43,6 \%)$ \\
$\begin{array}{c}\text { Grupo controle } \\
(\mathrm{n}=30)\end{array}$ & $5(16,7 \%)$ & $25(83,3 \%)$ \\
\hline
\end{tabular}

Hamilton para depressão encontramos valores elevados (entre 20 e 30 ) em 10 pacientes $(16,13 \%)$. Quando foram aplicados os critérios de DSM III$R, 9$ pacientes $(14,5 \%)$ classificaram-se como ansiedade, $8(12,9 \%)$ como distimia, $15(24,2 \%)$ como depressão ( 5 com depressão maior) e apenas $2(3,2 \%)$ como demência.

Dos 30 pacientes do grupo controle, 5 $(16,7 \%)$ apresentaram alterações de EEG caracterizadas pela presença de ondas de 5 a $15 \mathrm{~Hz}$, de localização difusa e bilateral, com predomínio fronto-temporal ou temporal, sem modificar-se com a fotoestimulação intermitente. $O$ mini-mental de todos os pacientes-controle encontrava-se com valores acima de 24 . Não encontramos qualquer distúrbio aplicando-se os critérios do DSM III-R e/ou a escala de Hamilton para depressão.

Comparando-se os achados de EEG dos pacientes parkinsonianos e do grupo controle (Tabela1) e aplicando-se o teste do qui quadrado, verificamos ser estatisticamente significativa a diferença entre os dois grupos quanto às alterações de EEG ( $p<0,001)$.

Comparando-se ainda os achados de EEG dos pacientes parkinsonianos, com e sem alterações psíquicas e aplicando-se o teste do qui quadrado, verificamos não existir associação entre esses atributos $(p=0,357)$, o mesmo ocorrendo ao compararmos os parkinsonianos com e sem alterações psíquicas com as diferentes regiões de acomentimento ao EEG ( $\mathrm{p}=0,126)$ (Tabela 2). A correlação entre cada tipo de distúrbio psíquico e as alteraçōes de EEG pode ser observada na Tabela 3.

Tabela 2. Alteraçōes nos EEG dos pacientes parkinsonianos com e sem distúrbios psiquicos.

\begin{tabular}{ccccc}
\hline \multirow{2}{*}{$\begin{array}{c}\text { Pacientes parkinsonianos } \\
(\mathrm{n}=62)\end{array}$} & $\begin{array}{c}\text { Eletrencefalograma } \\
\text { e bilaterais }\end{array}$ & $\begin{array}{c}\text { Alterações } \\
\text { fronto-temporais }\end{array}$ & $\begin{array}{c}\text { Alterações } \\
\text { temporais }\end{array}$ & Normal \\
\hline $\begin{array}{c}\text { com distúrbios psíquicos } \\
(\mathrm{n}=34)\end{array}$ & 13 & 1 & 3 & 17 \\
sem distúrbios psiquicos & $(38,2 \%)$ & $(3,0 \%)$ & $(8,8 \%)$ & $(50,0 \%)$ \\
$(\mathrm{n}=28)$ & 10 & 6 & 2 & 10 \\
& $(35,7 \%)$ & $(21,5 \%)$ & $(7,1 \%)$ & $(35,7 \%)$ \\
\hline
\end{tabular}

Tabela 3. Achados eletrencefalograficos nos diferentes distürbios psiquicos nos pacientes parkinsonianos.

\begin{tabular}{lcccc}
\hline \multirow{2}{*}{$\begin{array}{c}\text { Pacientes } \\
\text { parkinsonianos } \\
(n=34)\end{array}$} & $\begin{array}{c}\text { Alteraçōes difusas } \\
\text { e bilaterais }\end{array}$ & $\begin{array}{c}\text { Alterações } \\
\text { fronto-temporais }\end{array}$ & $\begin{array}{c}\text { Alterações } \\
\text { temporais }\end{array}$ & Normal \\
\hline \hline Ansiedade $(n=9)$ & $1(11,1 \%)$ & $3(33,3 \%)$ & $1(11,1 \%)$ & $4(44,5 \%)$ \\
Distimia $(n=8)$ & $1(12,5 \%)$ & - & $2(25,0 \%)$ & $5(62,5 \%)$ \\
Depressão $(n=15)$ & $4(26,7 \%)$ & $3(20,0 \%)$ & - & $8(53,3 \%)$ \\
Demencia $(n=2)$ & $1(50,0 \%)$ & $1(50,0 \%)$ & - & - \\
\hline
\end{tabular}




\section{COMENTÁRIOS}

Estudos anteriores fazem referência à ação de drogas dopaminérgicas sobre a atividade cortical, interferindo portanto com os resultados do $\mathrm{EEG}^{4,10}$. Assim sendo, nesses estudos a medicaçāo era suspensa 72 horas antes da realização do exame. Como no nosso estudo não foi possível manter esse período de supressão da droga, optamos por suspender a medicação 24 horas antes do exame, período bem aceito pelos pacientes.

Em nosso estudo as alterações do EEG encontradas tanto no grupo parkinsoniano como no grupo controle nāo diferiram muito quanto às suas características, sendo no primeiro grupo a porcentagem de EEG alterados $(56,4 \%)$ superior à do grupo controle $(16,7 \%)$. Essas alterações de EEG, difusas e lentas porém com predomínio fronto-temporal, fazem-nos deduzir que o comprometimento do cortex cerebral é mais evidente nos pacientes parkinsonianos, independentemente de apresentarem ou não distúrbios psíquicos.

Hughes descreveu alterações de EEG em $40 \%$ dos pacientes parkinsonianos, caracterizadas por ritmo de base lento, ondas lentas difusas e ausência de atividade epileptiforme $e^{5}$. Gautier e Quesney analisando o EEG de 25 pacientes com doença de Parkinson idiopática encontraram traçados anormais em 55\% dos casos,caracterizados por atividade lenta posterior e/ou ondas lentas difusas ${ }^{4}$. Garcia Mas e col. analisaram o EEG de pacientes parkinsonianos com e sem deteriorização cortical ${ }^{3}$. Eles concluiram que existem dois padrōes eletrofisiológicos na doença de Parkinson. Um deles corresponderia ao padrão observado na doença de Alzheimer, caracterizado por ondas teta e delta difusas superpostas por descargas de ondas lentas ritmadas intermitentes, e o outro reforçaria a hipotese de comprometimento dos lobos frontais como fundamento para as alterações cognitivas encontradas na doença de Parkinson sem comprometimento cortical.

O tempo de doença desses pacientes era de 1 a 20 anos de evolução; entretanto, nāo encontramos correlação entre a duração e a gravidade do quadro clínico, bem como entre o tempo de doença e a intensidade das alterações cognitivas encontradas. Assim, aqueles pacientes com depressão e/ou ansiedade não tinham quadro parkinsoniano mais severo, o mesmo sendo observado naqueles pacientes com depressão que não apresentavam os menores valores de mini-mental.

Apesar de haver relatos de que a depressão na doença de Parkinson possa ser acompanhada de comprometimento intelectual ${ }^{8}$, não encontramos em nosso estudo uma relação entre a gravidade da depressão e a severidade do comprometimento intelectual.

A depressão é sintoma muito comum em pacientes com doenças crônicas como a doença de Parkinson e nesses pacientes é observada entre $39 \%$ e $90 \%$ dos casos ${ }^{10,14}$. Alguns autores acreditam haver um mesmo substrato anatômico para a depressão e a doença de Parkinson. No entanto, não observamos relaçāo entre a intensidade dos distúrbios psíquicos, em especial a depressão, e a severidade do parkinsonismo, de acordo também com os achados de outros autores ${ }^{8-10}$. A diminuição da dopamina e as alterações degenerativas na substância negra e locus ceruleus não devem ser a causa da depressão e das alteraçōes cognitivas encontradas, porque nem todos os pacientes parkinsonianos apresentavam esses sintomas.

Encontramos apenas 2 casos de demência $(3,2 \%)$ entre os pacientes parkinsonianos avaliados em nosso estudo. Infelizmente, este achado não é suficiente para que possamos analisar esse distúrbio na doença de Parkinson, devido ao reduzido número de casos. No entanto, estudos realizados mostram que pacientes mais idosos tendem a desenvolver demência; as manifestações motoras surgem mais tardiamente e evoluem rapidamente para a incapacidade física, ocorrendo pouca resposta ao tratamento com levodopa e maior incidência de efeitos colaterais ${ }^{12,18}$.

Mais recentemente, a tomografia por emissão de positrons (PET) tem demonstrado os padrões de interrupção dos sistemas neurotransmissores dopaminérgico e outros nos pacientes parkinsonianos, 
assim como as anormalidades do metabolismo cerebral regional e do fluxo sanguíneo². Com observações desse tipo, poderíamos avaliar melhor as anormalidades encontradas em pacientes parkinsonianos com e sem comprometimento psíquico.

Agradecimento - Ao professor Adriano Caldeira de Araújo, Professor Adjunto do Instituto de Biologia da UERJ, por sua colaboração na análise estatística.

\section{REFERENCIAS}

1. Bieliauskas LA, Klawans HL, Glantz RH. Depression and cognitive changes in Parkinson's disease: a review. Adv Neurol 1986, 45: 437-438.

2. Brooks DJ. PET studies on the early and differential diagnosis of Parkinson's disease. Neurology 1993, 43 (Suppl. 6): S6-S16.

3. Garcia-Mas A, Far AR, Bennasar MR, Llinas J, Mir JR. Afectación frontal y deterioro cortical y subcortical en la enfermedad de Parkinson valorado mediante eletroencefalografia cuantificada. Arch Neurobiol 1991, 54: 303-310.

4. Gautier S, Quesney LF. Assesment of cortical dopaminergic activity in Parkinson's disease by EEG. Adv Neurol 1984, 40: 399-402.

5. Hughes HR. The electroencephalogram in Parkinsonism. J Neurosurg 1966, 24: 369-376.

6. Javoy-Agid F, Agid Y. Is the mesocortical dopaminergic system involved in Parkinson's disease? Neurology 1980, 30: 1326-1330.

7. Lieberman A, Dziatolowski M, Coopersmith M et al. Dementia in Parkinson's disease. Ann Neurol 1979, 6: 355-359.

8. Mayex R, Stern Y, Rosen J, Leventhal J. Depression, intelectual impairment and Parkinson's disease. Neurology 1981, 31: 645-650.

9. Mayeux R. Depression and dementia in Parkinson's disease. In Marsden CD, Fahn S (eds). Movement disorders. London: Butterworth, 1982, p 75-95.

10. Mayeux R, Stern Y, Cote L, Williams JBW. Altered serotonin metabolism in depressed patients with Parkinson's disease. Neurology 1984, 34: 642-646.

11. Mayeux R, Stern Y, Williams JBW, Sano M, Cote L. Depression and Parkinson disease. Adv Neurol 1986, 45: 451-455.

12. Mayeux R, Stern Y, Rosenstein R, Marder K, Hauser A, Cote L, Fahn S. An estimate of the prevalence of dementia in idiopathic Parkinson's disease. Arch Neurol 1988, 45: 260-262.

13. McCarthy R, Gresty M, Findley LJ. Parkinson's disease and dementia. Lancet 1985, 1: 407.

14. Mindham RHS. Psychiatric symptoms in parkinsonism. J Neurol Neurosurg Psychiatry 1970, 33: 188-191.

15. Mjones SH. Paralysis agitans: clinical and genetic study. Acta Psychiatr Neurol 1949, (Suppl) 54: 1-195.

16. Oyebode JR, Barker WA, Blessed G, Dick DJ, Britton PG. Cognitive functioning in PD in relation to prevalence of dementia and psychiatric disorders. Br J Psychiatry 1986, 149: 720-725.

17. Pollock $M$, Homabrook RW. The prevalence, natural history and dementia of Parkinson's disease. Brain 1966, 89: 429-448.

18. Rajput AH, Offord KP, Beard CM et al. A case-control sutdy of smoking habits, dementia and other illnesses in idiopathic Parkinson's disease. Neurology 1987, 37: 226-232.

19. Robins AA. Depression in patients with parkinsonism. Br J Psychiatry 1976, 128: 141-145.

20. Starkstein SE, Mayberg HS, Leiguarda R, Preziosi TJ, Robinson RG. A prospective longitudinal study of depression, cognitive declive, and physical impairments in patients with Parkinson's disease. J Neurol Neurosurg Psychiatry 1992, 55: 377-382.

21. Todes CJ. Idiopathic PD and depression: a psychosomatic view. J Neurol Neurosurg Psychiatry 1984, 47: 298-301.

22. Torack RM, Morris JC. The association of ventral tegmental area histopathology with adult dementia. Arch Neurol 1988, 45: 497-501.

23. Tune L et al. Familial manic depressive illness and familial PD. Johns Hopkins Med J 1982, 151: 65-70. 